\title{
Comparison of the effect of rosuvastatin versus rosuvastatin/ezetimibe on markers of inflammation in patients with acute myocardial infarction
}

\author{
YIZHI REN ${ }^{1}$, HAO ZHU ${ }^{1,2}$, ZHONGGUO FAN $^{1}$, YALI GAO $^{1}$ and NAILIANG TIAN ${ }^{1,2}$ \\ ${ }^{1}$ Department of Cardiology, Nanjing First Hospital, Nanjing Medical University; \\ ${ }^{2}$ Department of Cardiology, Nanjing Heart Center, Nanjing, Jiangsu 210006, P.R. China
}

Received October 5, 2016; Accepted June 22, 2017

DOI: $10.3892 / \mathrm{etm} .2017 .5175$

\begin{abstract}
Statins lower low-density lipoprotein cholesterol (LDL-C) and high-sensitivity C-reactive protein (hsCRP), and the addition of ezetimibe to statins further reduces LDL-C and hsCRP. Lipoprotein-associated phospholipase A2 (Lp-PLA2) is a potentially important pathogenic factor participating in the progression of atherosclerosis. The aim of current study was to investigate how the addition of ezetimibe to rosuvastatin treatment affects reductions in LDL-C, hsCRP and Lp-PLA2 in patients with acute myocardial infarction (AMI). A total of 135 patients were enrolled in the study within $24 \mathrm{~h}$ of AMI, and were randomized to receive $10 \mathrm{mg}$ rosuvastatin or $10 \mathrm{mg}$ rosuvastatin plus $10 \mathrm{mg}$ ezetimibe daily. HsCRP, Lp-PLA2, total cholesterol (TC), triglycerides (TG), LDL-C and high-density lipoprotein cholesterol (HDL-C) were determined at baseline and after 1, 3, 6 and 12 months of treatment. The addition of ezetimibe to rosuvastatin led to greater reduction of LDL-C compared with rosuvastatin monotherapy (from 3.00 to $1.19 \mathrm{mmol} / 1$ vs. 2.93 to $1.49 \mathrm{mmol} / 1$, respectively; $\mathrm{P}<0.05)$, as well as reduced levels of hsCRP (from 5.15 to $0.68 \mathrm{mg} / \mathrm{l} \mathrm{vs}$. 4.33 to $1.49 \mathrm{mg} / \mathrm{l}$, respectively; $\mathrm{P}<0.05$ ) and Lp-PLA2 (from 333.13 to $79.07 \mathrm{mg} / \mathrm{l}$ vs. 327.95 to $123.62 \mathrm{mg} / \mathrm{l}$, respectively; $\mathrm{P}<0.05)$. A positive association was identified between reductions of Lp-PLA2 and the changes of LDL-C $(r=0.367$; $\mathrm{P}=0.002)$. However, no significant correlation was observed between changes in Lp-PLA2 and hsCRP $(r=0.264$; $P=0.512)$. The values of hsCRP and Lp-PLA2 appeared to increase during the first week after randomization, but dropped steeply to a lower level and remained stable thereafter. In conclusion, the addition of ezetimibe to rosuvastatin was demonstrated to
\end{abstract}

Correspondence to: Dr Nailiang Tian, Department of Cardiology, Nanjing First Hospital, Nanjing Medical University, 68 Changle Road, Nanjing, Jiangsu 210006, P.R. China

E-mail: tiannailiang01@163.com

Key words: rosuvastatin, ezetimibe, LDL-C, hsCRP, Lp-PLA2, AMI further reduce LDL-C, hsCRP and Lp-PLA2 compared with rosuvastatin monotherapy in patients with AMI.

\section{Introduction}

Atherosclerosis is a complex chronic inflammatory condition that involves an excessive inflammatory response and lipid accumulation (1). Reduction of low-density lipoprotein (LDL) levels by treatment with a statin is an established method for the prevention of cardiovascular disease. Statins are able to reduce mortality and morbidity by lowering blood lipid levels and inhibiting the inflammatory response. Previous studies have indicated that statin use is associated with a reduction in the levels of serum high-sensitivity C-reactive protein (hsCRP) that occurs independently of the reduction in LDL-cholesterol (LDL-C) (2-4).

Ezetimibe is a cholesterol absorption inhibitor that prevents the absorption of dietary and biliary cholesterol from the small intestine. Previous studies indicate that when used in combination with statin therapy, ezetimibe produces a further $23-24 \%$ reduction in LDL-C and $9-10 \%$ reduction in hsCRP compared with statin therapy alone (5-8). The Improved Reduction of Outcomes: Vytorin Efficacy International Trial (IMPROVE-IT) demonstrated that a significantly greater proportion of patients treated with ezetimibe plus simvastatin met the specified targets of LDL-C $<70 \mathrm{mg} / \mathrm{dl}$ and hsCRP $<2 \mathrm{mg} / \mathrm{l}$ when compared with simvastatin monotherapy (9). In that trial, achievement of these two targets was associated with improved clinical outcomes following multivariable adjustment, which may indicate a strategy in which the lipoprotein and inflammatory profile are monitored (9).

Lipoprotein-associated phospholipase A2 (Lp-PLA2) is excreted predominantly from atherosclerotic plaques by macrophages and neutrophils and subsequently circulates in the blood stream (10). It has been demonstrated that Lp-PLA2 is a potentially important pathogenic factor participating in the progression of atherosclerosis (11). In an animal model of hyperlipidemia and hyperglycemia, an Lp-PLA2 inhibitor reduced macrophage accumulation, and diminished the necrotic lipid-core volume and fibrous cap of coronary atherosclerotic plaques (12). Furthermore, the mass and activity 


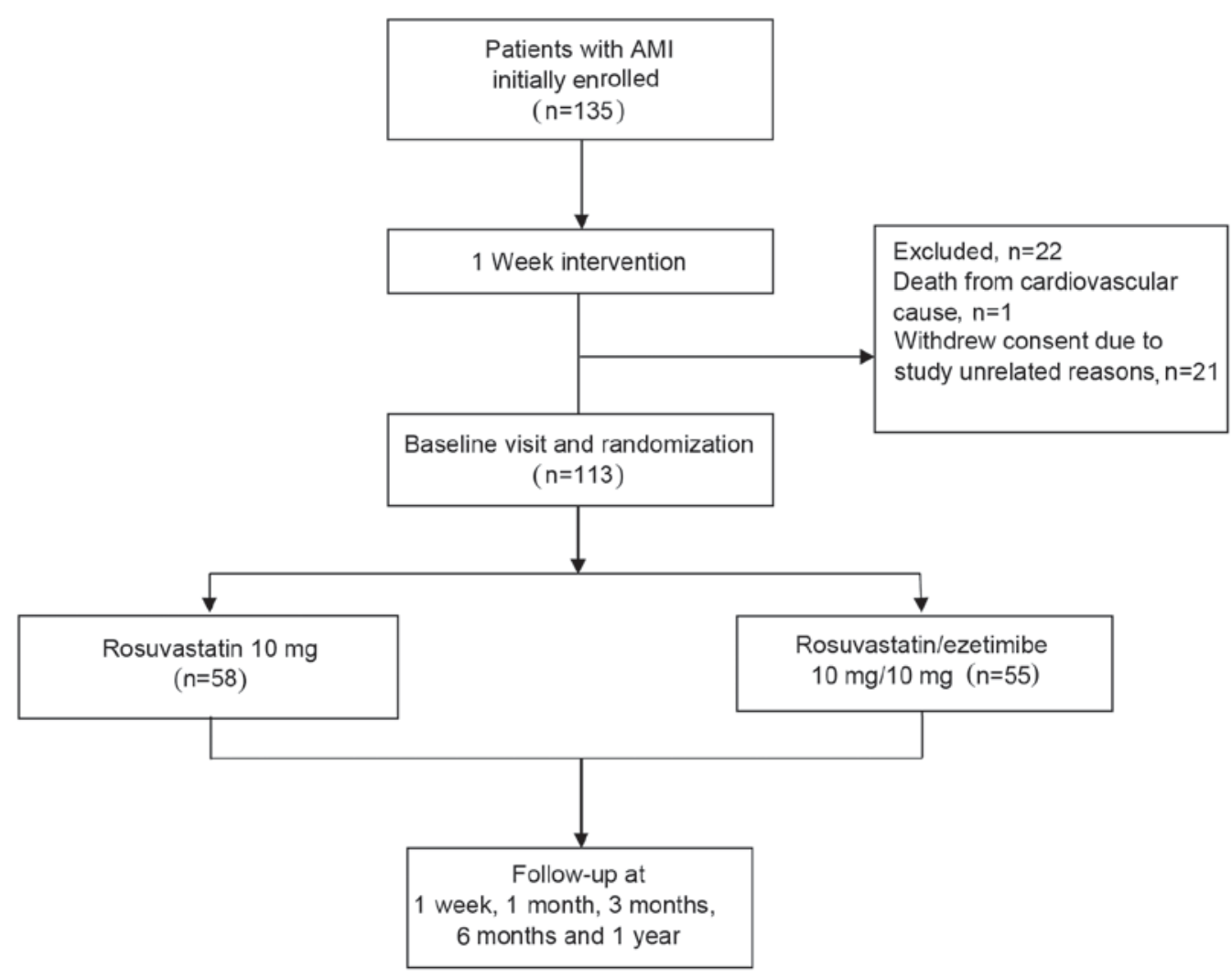

Figure 1. Flow diagram of study participants and their treatments. AMI, acute myocardial infarction.

of Lp-PLA2 is indicated to be positively correlated with an increased risk of coronary artery disease and stroke (13). Statins also have been shown to reduce Lp-PLA2 levels by up to $33 \%(14,15)$. The Long-term Intervention with Pravastatin in Ischemic Disease (LIPID) study demonstrated that the reduction in Lp-PLA2 activity during the first year of treatment was a highly significant predictor of coronary heart disease events, independent of any change in LDL-C, and may account for over half of the benefits of pravastatin (16).

Taking previous studies into consideration, the present study sought to investigate: i) Whether the addition of ezetimibe to rosuvastatin would further reduce LDL-C and hsCRP as compared with rosuvastatin monotherapy; ii) whether the addition of ezetimibe to rosuvastatin would further reduce Lp-PLA2 in comparison with rosuvastatin alone and iii) if so, whether the reduction of Lp-PLA2 differed for treatment with ezetimibe and rosuvastatin vs. rosuvastatin alone across prespecified subgroups; iv) the possible alterations of Lp-PLA2 and hsCRP levels during the development of acute myocardial infarction (AMI) and their relationship with LDL-C and other lipid parameters.

\section{Materials and methods}

Study population and procedures. All the subjects in the study were inpatients at the Department of Cardiology, Nanjing First Hospital Affiliated with Nanjing Medical University (Nanjing, China) from January 2015 to June 2016. Inclusion criteria were that patients aged within the range of 18 to 80 years were eligible if hospitalized within the preceding $24 \mathrm{~h}$ for
AMI, including ST-segment elevation myocardial infarction (STEMI) with or without ST-segment elevation myocardial infarction (NSTEMI). STEMI was defined as an AMI with dynamic changes in the electrocardiogram and at least one instance of elevated levels of cardiac enzymes or myocardial necrosis biomarkers, defined as total creatine phosphokinase or creatine kinase major basic fraction $>2$-fold the upper limit of the normal range and/or positive troponin I or troponin $\mathrm{T}$. The exclusion criteria were: i) Contraindications for the intervention; ii) statin use was contraindicated, for example, due to the patient having active hepatitis or being allergic to statins; iii) severe cardiac dysfunction (Killip class III or IV); iv) severe renal insufficiency; and v) other comorbidities, including infection, systemic immune diseases, pericarditis and malicious tumor.

Initially, 135 patients (age: $62.7 \pm 3.8$ years; male: female, 109:26) were enrolled (Fig. 1). Following 1 week of the intervention, 113 patients (age: 59.0 \pm 2.0 years; male: female, 94:19) continued to meet the inclusion criteria and were randomly divided into two groups: Ezetimibe $(10 \mathrm{mg}$; Merck KGaA, Darmstadt, Germany) plus rosuvastatin (10 mg; IPR Pharmaceuticals, Inc., Canovanas, PR, USA) group (combination group; $n=5)$ and rosuvastatin $(10 \mathrm{mg})$ group $(n=58)$. Randomization was performed by means of a computer-generated sequence of random numbers. Participants were instructed to take tablets once daily in the evening and to pay attention to the side effects of rosuvastatin and ezetimibe. The patients' age, sex, medical history, smoking history, echocardiography parameters, blood lipid levels, Lp-PLA2 and hsCRP were recorded at admission within the first $24 \mathrm{~h}$ after the onset of 
Table I. Baseline clinical characteristics.

\begin{tabular}{lccc}
\hline Characteristic & R10 (n=58) & $\begin{array}{c}\text { R10+E10 } \\
(\mathrm{n}=55)\end{array}$ & P-value \\
\hline Males, n (\%) & $46(79.3)$ & $48(87.3)$ & 0.258 \\
Age, years & $60.7 \pm 1.3$ & $57.3 \pm 1.5$ & 0.123 \\
Current smoker, n (\%) & $38(65.5)$ & $39(70.9)$ & 0.539 \\
Hypertension, n (\%) & $35(60.3)$ & $31(56.4)$ & 0.668 \\
Diabetes mellitus, n (\%) & $10(17.2)$ & $10(18.2)$ & 0.896 \\
Dyslipidemia, n (\%) & $22(37.9)$ & $20(36.4)$ & 0.863 \\
Previous MI, n (\%) & $1(1.7)$ & $2(3.6)$ & 0.527 \\
Previous PCI, n (\%) & $3(5.3)$ & $5(9.1)$ & 0.432 \\
Previous medication, & & & \\
n (\%) & & & \\
Statins & $6(10.5)$ & $5(9.1)$ & 0.799 \\
ASA & $10(17.5)$ & $12(21.8)$ & 0.569 \\
Thienopyridine & $3(5.3)$ & $5(9.1)$ & 0.432 \\
CCB & $11(19.3)$ & $5(9.1)$ & 0.123 \\
Diuretics & $3(5.3)$ & $4(7.3)$ & 0.660 \\
ACEI/ARB & $4(7.0)$ & $9(16.4)$ & 0.123 \\
\hline
\end{tabular}

$\mathrm{R} 10,10 \mathrm{mg}$ rosuvastatin; $\mathrm{R} 10+\mathrm{E} 10,10 \mathrm{mg}$ rosuvastatin plus $10 \mathrm{mg}$ ezetimibe; MI, myocardial infarction; PCI, percutaneous coronary intervention; ASA, aspirin; CCB, calcium channel blocker.

AMI. All patients received treatment according to common guidelines, including appropriate use of antiplatelet agents, anticoagulants, statins, $\beta$-blockers and revascularization. The therapies administered were identical in the two groups (Fig. 1). This study was approved by the Ethics Committee of Nanjing First Hospital. All subjects provided written informed consent.

Clinical data collection. Visits to hospital took place at baseline, and at 1, 3 and 12 months after drug treatment commenced. Blood samples were obtained at each visit and echocardiography reexamination was arranged at 12 months. Serum levels of total cholesterol (TC), triglycerides (TG), high-density lipoprotein cholesterol (HDL-C), LDL-C, hsCRP and Lp-PLA2 were measured using standard enzymatic methods in the hospital laboratory as previously described (17).

Statistical analysis. All data were analyzed using SPSS 24.0 software (IBM Corp., Armonk, NY, USA). Numerical data are expressed as n (\%). Measurement data are expressed as the mean \pm standard deviation. Means of the two groups were compared using an independent sample t-test. Means in a group prior to and following treatment were compared using a paired t-test. Pearson correlation coefficients were used to investigate the correlation of changes in plasma biomarkers and lipids from baseline to 12 months (bivariate correlation analysis). The results were presented using PRISM version 6.0 (GraphPad Software, Inc., La Jolla, CA, USA). P<0.05 (two-tailed) was considered to indicate a statistically significant result.

\section{Results}

Demographic characteristics. Initially, 135 patients were enrolled (Fig. 1). Following 1 week of intervention, 21 patients withdrew consent and were excluded from the study. A total of 113 patients continued to participate and were randomized to receive either $10 \mathrm{mg}$ rosuvastatin $(\mathrm{n}=58)$ or the combination of $10 \mathrm{mg}$ rosuvastatin plus $10 \mathrm{mg}$ ezetimibe $(\mathrm{n}=55)$. All randomized subjects completed the study. Baseline characteristics (Table I), lipid and biomarkers (Table II) were generally consistent in the two treatment groups and were not significantly different.

Effect on lipids. All lipid parameters were obtained at 1 week, and 1, 3, 6 and 12 month, after randomization (Table II and Fig. 2) and changes from baseline were evaluated (Table III and Fig. 3). Compared with baseline, significant reductions were obtained in the two groups for TC, LDL-C and TG over the duration of the study (Table II). During the study duration, marked increases in HDL-C from baseline were also observed in the two groups, although there was a slight reduction at 1 week (Table II). During the 12 months of observation, LDL-C significantly decreased from 3.00 to $1.19 \mathrm{mmol} / \mathrm{l}$ in the combination group and from 2.93 to $1.49 \mathrm{mmol} / 1$ in the rosuvastatin group. The addition of ezetimibe to rosuvastatin resulted in further reductions in mean LDL-C over the study duration in comparison with rosuvastatin alone (Fig. 2A). Net changes from baseline in TC and LDL-C were significantly greater in the combination group compared with the rosuvastatin group; however, no significant difference in the change of TG levels was observed between the two groups (Table III; Fig. 3A).

Effect on inflammatory markers. Inflammatory markers, hsCRP and Lp-PLA2, were evaluated at 1 week, and 1, 3, 6 and 12 months, after randomization. Compared with baseline, significant reductions were obtained in the two groups for hsCRP and Lp-PLA2 over the study duration (Table II). The values of hsCRP and Lp-PLA2 increased substantially 1 week after randomization, and then dropped steeply within the next 3 weeks, after which the values remained stable (Fig. 2B and C). During the 12 months of observation, hsCRP decreased significantly from 5.15 to $0.68 \mathrm{mg} / \mathrm{l}$ in the combination group and from 4.33 to $1.49 \mathrm{mg} / \mathrm{l}$ in the rosuvastatin alone group $(\mathrm{P}=0.01$; Table II). The addition of ezetimibe to rosuvastatin resulted in a further reduction in mean hsCRP over the study duration in comparison with rosuvastatin alone (Fig. 2B). Similarly, during the 12 months of observation, Lp-PLA2 decreased significantly from 333.13 to $79.07 \mathrm{mg} / \mathrm{l}$ in the combination group and from 327.95 to $123.62 \mathrm{mg} / \mathrm{l}$ in the rosuvastatin alone group $(\mathrm{P}=0.04$; Table II). The addition of ezetimibe to rosuvastatin resulted in a further reduction in mean Lp-PLA2 over the study duration in comparison with rosuvastatin monotherapy (Fig. 2C). Net changes from baseline in hsCRP and Lp-PLA2 were significantly greater in the combination group compared with the rosuvastatin alone group (Table III; Fig. 3B and C). Treatment effects for each of the treatments were similar for net changes from baseline to 12 months in Lp-PLA2 across prespecified subgroup categories of age, gender, smoking and diabetic status (Fig. 4). 


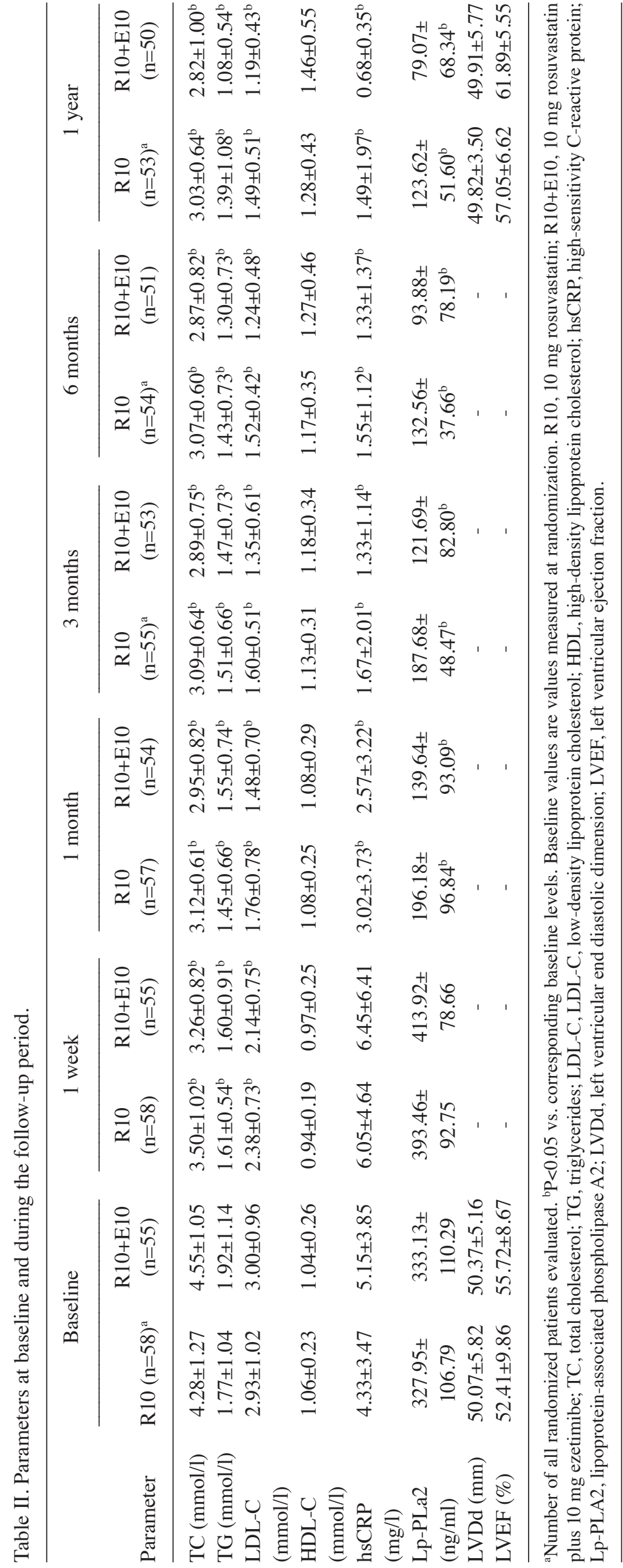


A

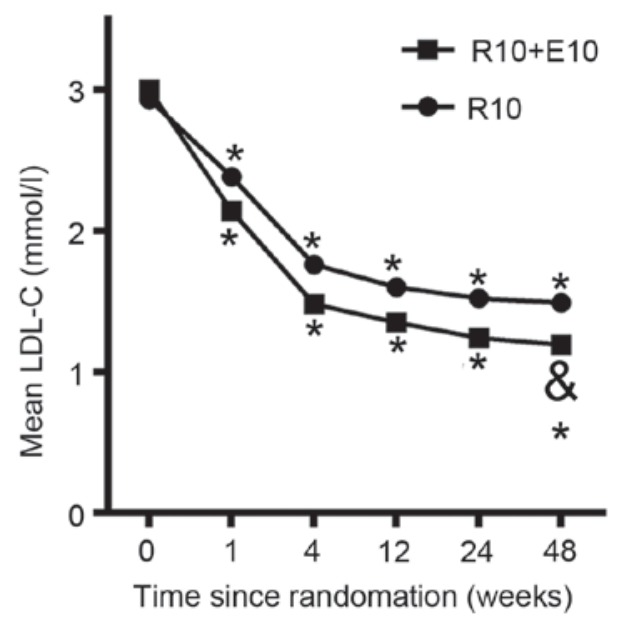

B

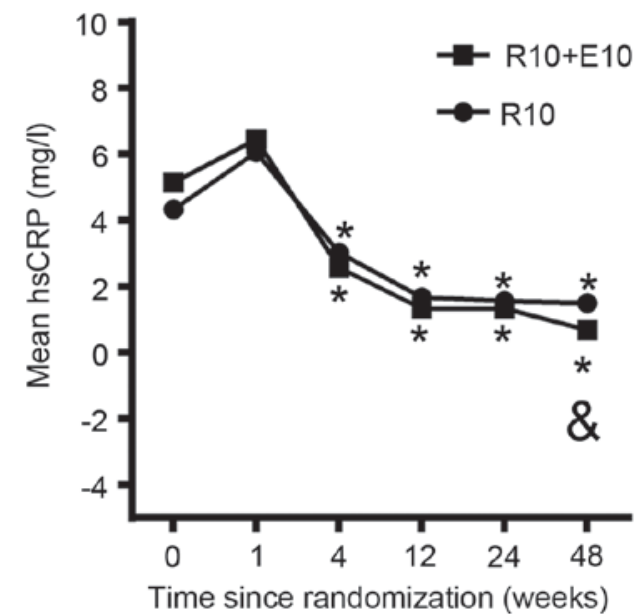

C

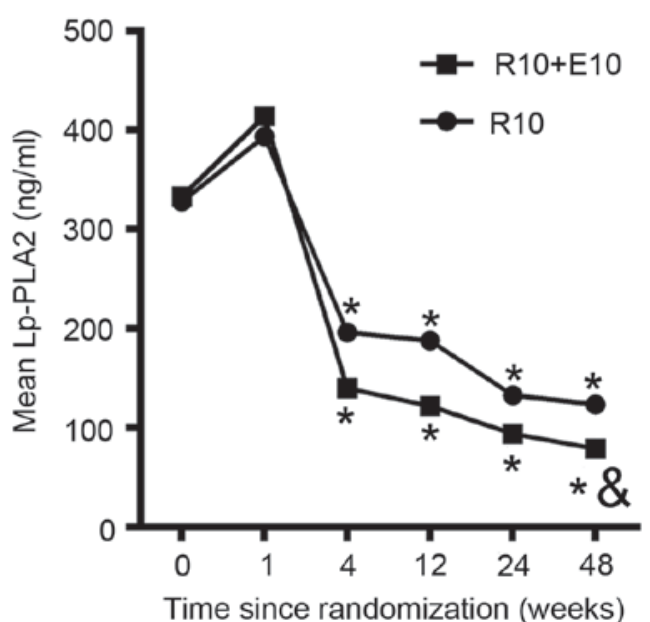

Figure 2. LDL-C, Lp-PLA2 and hsCRP levels over time following randomized treatment. Plots of mean (A) LDL-C, (B) hsCRP and (C) Lp-PLA2 over time. LDL-C, low-density lipoprotein cholesterol; hsCRP, high-sensitivity C-reactive protein; Lp-PLA2, lipoprotein-associated phospholipase A2; R10, $10 \mathrm{mg}$ rosuvastatin; R10+E10, $10 \mathrm{mg}$ rosuvastatin plus $10 \mathrm{mg}$ ezetimibe. Numerical values and standard deviations for the results in these plots are shown in Table II. ${ }^{*} \mathrm{P}<0.05$ vs. corresponding baseline levels; ${ }^{\circledR} \mathrm{P}<0.05$ vs. $\mathrm{R} 10$ group.

Correlation between lipids and inflammatory markers. Table IV shows correlations between lipids and inflammatory markers. Although LDL-C and Lp-PLA2 levels decreased from baseline, the correlation in achieved net changes from baseline to 12 months for LDL-C and Lp-PLA2 values was weak in all subjects $(\mathrm{r}=0.367 ; \mathrm{P}=0.002)$ and not significant for the combination treatment and the rosuvastatin monotherapy respectively (data not shown). Furthermore, Lp-PLA2 did not correlate with hsCRP in all subjects combined $(r=0.264$; $\mathrm{P}=0.512$ ), nor in the combination treatment or rosuvastatin monotherapy groups respectively (date not shown). In addition, no significant correlation was identified between Lp-PLA2 and total cholesterol or HDL-C. Similarly, no significant correlation was observed between hsCRP and lipid values. Even though Lp-PLA2 had positive correlations with LDL-C, the correlations were weak.

Change of cardiac function. As shown in Tables II and III, left ventricular end diastolic dimension (LVDd) and left ventricular ejection fraction (LVEF) were measured at baseline and 12 months after randomization. Although there was greater reduction in LVDd and further increase in LVEF with the addition of ezetimibe to rosuvastatin in comparison with rosuvastatin alone, the differences were not found to be significant $(\mathrm{P}=0.616$ and $\mathrm{P}=0.882$, respectively).

\section{Discussion}

In the present study, the results suggested that the combined treatment led to a greater reduction of LDL-C, as well as significantly reduced levels of hsCRP and Lp-PLA2 compared with rosuvastatin monotherapy. Moreover, it was found that the reduction of Lp-PLA2 had a positive correlation with the change of LDL-C, although the correlation was weak. In addition, no significant correlation was detected between changes in Lp-PLA2 and hsCRP. Although the addition of ezetimibe to the treatment resulted in a greater reduction of Lp-PLA2, the increases did not achieve statistical significance in prespecified subgroups. To clarify the alterations of Lp-PLA2 and hsCRP levels during the development of AMI, blood samples were obtained from the patients and assessed within the first $24 \mathrm{~h}$ of admission and patients had follow-up visits at 1 week, and 1, 3, 6, and 12 months thereafter. It was observed that the values of hsCRP and Lp-PLA2 appeared to increase at week 1 after randomization, then dropped steeply to a lower level and remain stable after that. The findings of the present study are novel in that they extend previous observations with statin therapy to non-statin-based therapeutic augmentation with ezetimibe, an agent with no observed safety concerns (18). In the analysis conducted in the present study, reductions in Lp-PLA2 were observed to be significantly greater with combination therapy compared with monotherapy. These results are consistent with previous studies that demonstrated the degree of Lp-PLA2 reduction with combination therapy was consistent with the extent of LDL-C lowering efficacy (19-22). Furthermore, it has been verified that the addition of ezetimibe to rosuvastatin or simvastatin provides additional reductions in Lp-PLA2 mass and activity in patients already receiving statin monotherapy (20). It was observed in the present study that the addition of ezetimibe to rosuvastatin further reduced LDL-C and hsCRP levels, as compared with the levels achieved using rosuvastatin monotherapy. These 


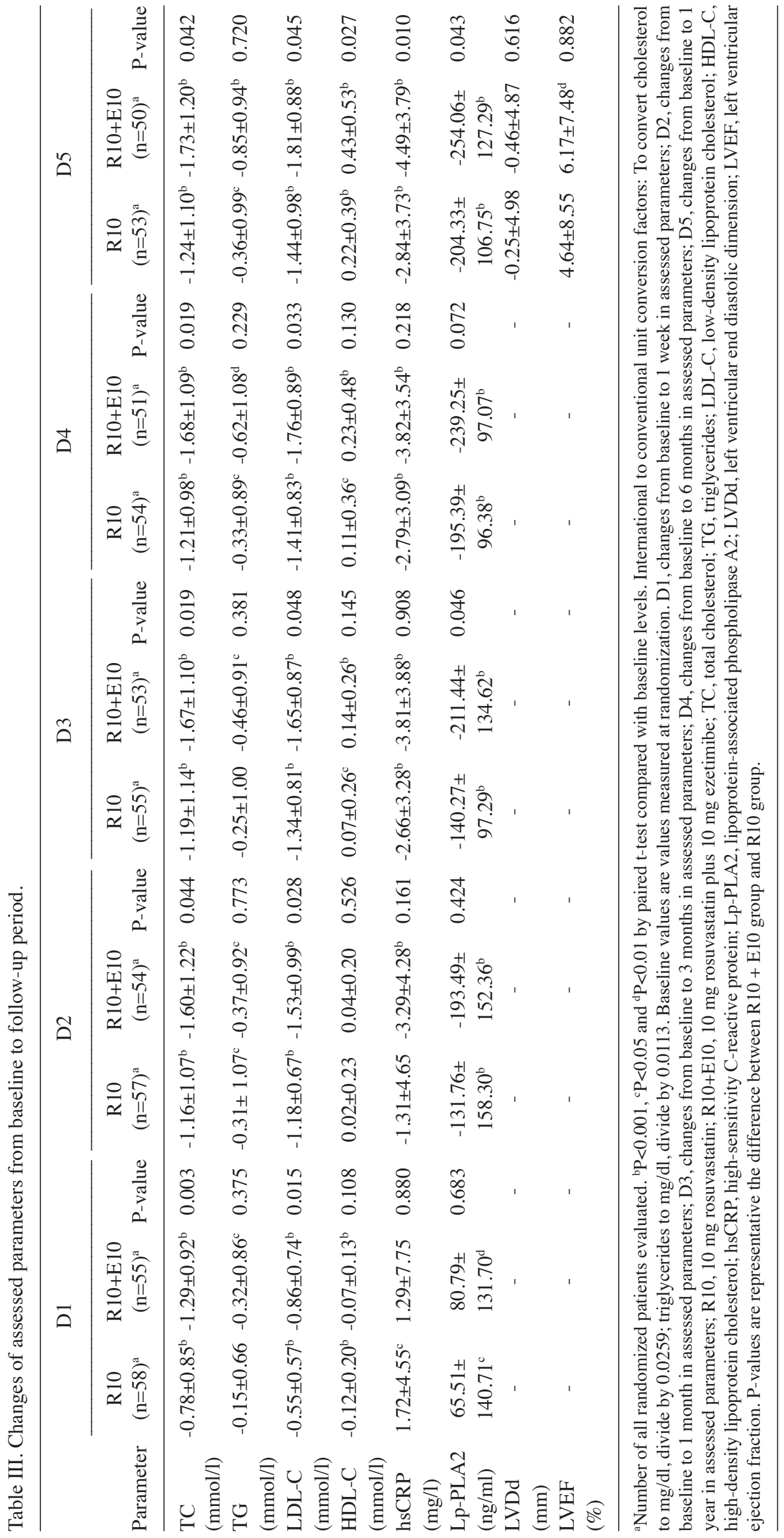


A
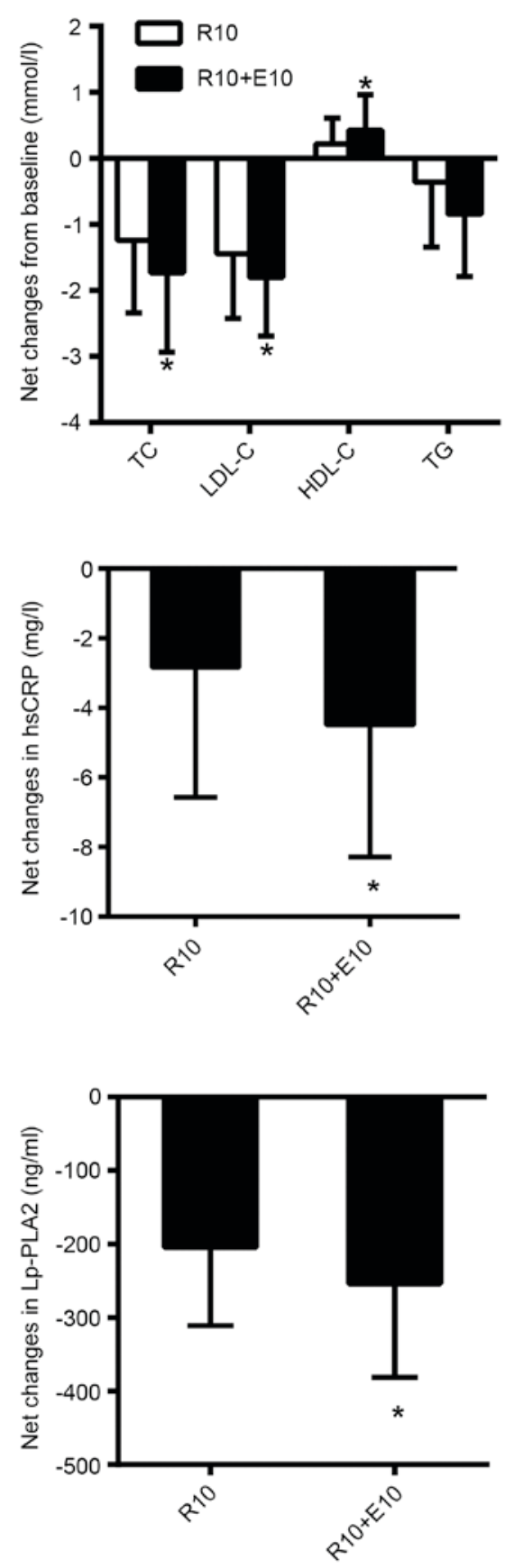

Table IV. Correlations between net changes from baseline to 12 months in inflammatory markers and lipids.

\begin{tabular}{lcccccc}
\hline & \multicolumn{2}{c}{$\begin{array}{c}\text { Change in } \\
\text { hsCRP }\end{array}$} & & \multicolumn{2}{c}{$\begin{array}{c}\text { Change in } \\
\text { Lp-PLA2 }\end{array}$} \\
\cline { 2 - 3 } \cline { 6 - 7 } Variables & $\mathrm{r}$ & P-value & & $\mathrm{r}$ & P-value \\
\cline { 2 - 3 } \cline { 5 - 7 } Change in Lp-PLA2 & 0.264 & 0.512 & & - & - \\
Change in hsCRP & - & - & & 0.264 & 0.512 \\
Change in TC & -0.173 & 0.115 & & 0.204 & 0.500 \\
Change in TG & -0.090 & 0.571 & & 0.243 & 0.790 \\
Change in LDL-C & -0.082 & 0.473 & & 0.367 & 0.002 \\
Change in HDL-C & -0.061 & 0.579 & -0.150 & 0.150 \\
\hline
\end{tabular}

hsCRP, high-sensitivity C-reactive protein; Lp-PLA2, lipoprotein-associated phospholipase A2; TC, total cholesterol; TG, triglycerides; LDL-C, low-density lipoprotein cholesterol; HDL-C, high-density lipoprotein cholesterol.

A

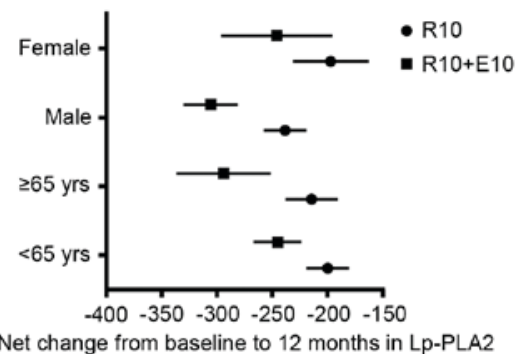
$(\mathrm{ng} / \mathrm{ml})$

B

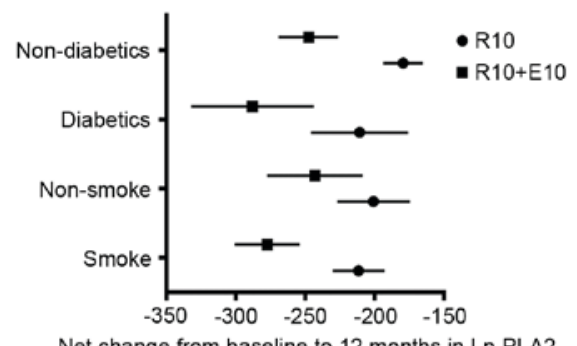

Net change from baseline to 12 months in Lp-PLA2 $(\mathrm{ng} / \mathrm{ml})$

C

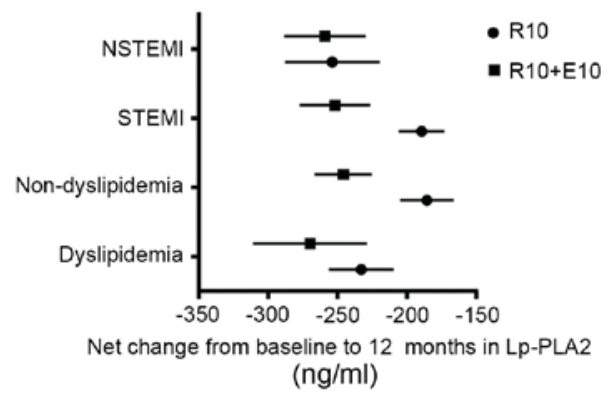

Figure 4. Net changes from baseline to 12 months in Lp-PLA2 for prespecified subgroups. The subgroups were (A) sex and age $\geq 65$ or $<65$ years, (B) diabetic status and smoking status, (C) STEMI or NSTEMI and dyslipidemia status. Lp-PLA2, lipoprotein-associated phospholipase A2; STEMI, ST-segment elevation myocardial infarction, NSTEMI, non-ST-segment elevation myocardial infarction; $\mathrm{R} 10,10 \mathrm{mg}$ rosuvastatin; $\mathrm{R} 10+\mathrm{E} 10,10 \mathrm{mg}$ rosuvastatin plus $10 \mathrm{mg}$ ezetimibe. 
mitogen-activated protein kinase pathway (23). Ezetimibe, rosuvastatin and fenofibrate monotherapy have been shown to primarily reduce the activity and mass of Lp-PLA2, which are associated with LDL lipoprotein subfractions via receptor-mediated uptake (24). Furthermore, it has also been reported that non-statin lipid-lowering therapies reduce Lp-PLA2 through a receptor-independent clearance mechanism and that Lp-PLA2 changes are weakly correlated with LDL-C changes, indicating that Lp-PLA2 reduction is only partly explained by LDL-C lowering (25). Likewise, in the present study, the results indicated that reductions in Lp-PLA2 with statins or combination therapy were proportionate to the extent of LDL-C lowering, along with weak correlations between reductions in Lp-PLA2 and changes of LDL-C.

The data regarding the correlation of Lp-PLA2 changes and hsCRP changes with statin or non-statin therapy obtained in the present study are in line with those in other reports. Ostadal et al reported that no association was observed between the alterations in the levels of Lp-PLA2 and $\mathrm{C}$-reactive protein (CRP; $r=0.06, \mathrm{P}=0.70)(26)$. Another study also reported a lack of correlation between Lp-PLA2 and CRP (27). In the Pravastatin Inflammation/CRP Study (PRINCE) study, a weak, but statistically significant correlation between changes in Lp-PLA2 levels and CRP change $(r=-0.13, P=0.05)$ was observed (15). While the reason for these results is unclear, data concerning markers of inflammation and Lp-PLA2 levels are conflicting. Some data suggest that pro-inflammatory-mediators, such as LPS, tumor necrosis factor (TNF)- $\alpha$ and interleukin (IL)-1, -6 and -8 , result in the downregulation of Lp-PLA2 by macrophages (28). However, the opposite observation was noted when TNF- $\alpha$ and IL-1 were administered to experimental animals (29).

Although the addition of ezetimibe to rosuvastatin resulted in a greater reduction of Lp-PLA2, the difference between the monotherapy and combination treatment did not achieve statistical significance in prespecified subgroups. Consistent with these results, a previous study reported that correlations between non-smokers and smokers and Lp-PLA2 levels were small and did not significantly affect the change in Lp-PLA2 when evaluated using multivariate analyses (15). However, as only 113 patients were examined in the present study, the results may have been due to chance. The results require confirmation in a larger study. However, these results possibly indicate that a high baseline Lp-PLA2 level may not be associated with a further reduction of Lp-PLA2 following treatment.

It was observed that the values of hsCRP and Lp-PLA2 increased at 1 week after randomization, and then dropped steeply to a lower level and remain stable thereafter. These results are consistent with those of previous studies, which have found that CRP exhibited a more marked increase during the first 24-96 $\mathrm{h}$ from symptom onset and markedly decreased over 30 days $(30,31)$. Notably, the transient elevation of CRP levels might indicate a propensity for a pronounced inflammatory response and is associated with increased mortality $(32,33)$. In contrast with the results of the present study, serum levels of Lp-PLA2 have been reported to decrease significantly following acute vascular events (median, 5 days; range 2-40 days), such as stroke and myocardial infarction (34). In the present study, baseline values of Lp-PLA2 were obtained and assessed within the first $24 \mathrm{~h}$ after the onset of symptoms. Therefore, it is possible that the baseline levels of Lp-PLA2 were obtained during the reduction phase following an acute event, and the high level of Lp-PLA2 observed at 1 week was a return toward the usual baseline levels that existed prior to the acute coronary syndrome.

The present study has several limitations, mostly attributable to the limited size of the study population. It may also be argued that the inclusion of the two types of AMI (ST elevation and non-ST elevation) may have increased the heterogeneity of the study group. As only 113 patients were enrolled in the study, the results may have been due to chance. Therefore, the results require confirmation in a larger study. Also, the study subjects were patients with AMI, so it is not known whether the results are applicable to other populations. Further studies with additional populations are needed.

In conclusion, the preset study confirmed that the addition of ezetimibe to rosuvastatin further reduced LDL-C and hsCRP, and also resulted in further reductions in Lp-PLA2, as compared with rosuvastatin monotherapy. The alterations in the levels of Lp-PLA2 were weakly but significantly correlated with LDL changes, which indicated that Lp-PLA2 reduction is only partly explained by LDL-C lowering. These findings provide evidence for the debate concerning the value of adjunctive LDL-C reduction in combination with a statin. In addition, reductions of Lp-PLA2 levels may be considered as target for the suppression of inflammation.

\section{References}

1. Ross R: The pathogenesis of atherosclerosis: A perspective for the 1990s. Nature 362: 801-809, 1993.

2. de Lemos JA, Blazing MA, Wiviott SD, Lewis EF, Fox KA White HD, Rouleau JL, Pedersen TR, Gardner LH, Mukherjee R, et al: Early intensive vs a delayed conservative simvastatin strategy in patients with acute coronary syndromes: Phase Z of the A to Z trial. JAMA 292: 1307-1316, 2004.

3. Ridker PM, Cannon CP, Morrow D, Rifai N, Rose LM, McCabe $\mathrm{CH}$, Pfeffer MA and Braunwald E; Pravastatin or Atorvastatin Evaluation and Infection Therapy-Thrombolysis in Myocardial Infarction 22 (PROVE IT-TIMI 22) Investigators: $\mathrm{C}$-reactive protein levels and outcomes after statin therapy. $\mathrm{N}$ Engl J Med 352: 20-28, 2005.

4. Ridker PM, Danielson E, Fonseca FA, Genest J, Gotto AM Jr, Kastelein JJ, Koenig W, Libby P, Lorenzatti AJ, MacFadyen JG, et al: Rosuvastatin to prevent vascular events in men and women with elevated C-reactive protein. $\mathrm{N}$ Engl J Med 359: 2195-2207, 2008.

5. Ballantyne CM, Blazing MA, King TR, Brady WE and Palmisano J: Efficacy and safety of ezetimibe co-administered with simvastatin compared with atorvastatin in adults with hypercholesterolemia. Am J Cardiol 93: 1487-1494, 2004.

6. Morrone D, Weintraub WS, Toth PP, Hanson ME, Lowe RS, Lin J, Shah AK and Tershakovec AM: Lipid-altering efficacy of ezetimibe plus statin and statin monotherapy and identification of factors associated with treatment response: A pooled analysis of over 21,000 subjects from 27 clinical trials. Atherosclerosis 223 : 251-261, 2012.

7. Pearson TA, Ballantyne CM, Veltri E, Shah A, Bird S, Lin J, Rosenberg E and Tershakovec AM: Pooled analyses of effects on C-reactive protein and low density lipoprotein cholesterol in placebo-controlled trials of ezetimibe monotherapy or ezetimibe added to baseline statin therapy. Am J Cardiol 103: 369-374, 2009.

8. Pearson T, Ballantyne C, Sisk C, Shah A, Veltri E and Maccubbin D: Comparison of effects of ezetimibe/simvastatin versus simvastatin versus atorvastatin in reducing $\mathrm{C}$-reactive protein and low-density lipoprotein cholesterol levels. Am J Cardiol 99: 1706-1713, 2007. 
9. Bohula EA, Giugliano RP, Cannon CP, Zhou J, Murphy SA, White JA, Tershakovec AM, Blazing MA and Braunwald E: Achievement of dual low-density lipoprotein cholesterol and high-sensitivity $\mathrm{C}$-reactive protein targets more frequent with the addition of ezetimibe to simvastatin and associated with better outcomes in IMPROVE-IT. Circulation 132: 1224-1233, 2015.

10. Macphee $\mathrm{CH}$, Nelson $\mathrm{J}$ and Zalewski A: Role of lipoprotein-associated phospholipase A2 in atherosclerosis and its potential as a therapeutic target. Curr Opin Pharmacol 6: 154-161, 2006

11. Ragab SM, Safan MA, Obeid OM and Sherief AS: Lipoprotein-associated phospholipase A2 (Lp-PLA2) and tumor necrosis factor-alpha (TNF- $\alpha$ ) and their relation to premature atherosclerosis in $\beta$-thalassemia children. Hematology 20 228-238, 2015

12. Wilensky RL, Shi Y, Mohler ER III, Hamamdzic D, Burgert ME, Li J, Postle A, Fenning RS, Bollinger JG, Hoffman BE, et al: Inhibition of lipoprotein-associated phospholipase $\mathrm{A} 2$ reduces complex coronary atherosclerotic plaque development. Nat Med 14: 1059-1066, 2008.

13. Lp-PLA(2) Studies Collaboration, Thompson A, Gao P, Orfei L, Watson S, Di Angelantonio E, Kaptoge S, Ballantyne C, Cannon CP, Criqui M, et al: Lipoprotein-associated phospholipase $\mathrm{A}(2)$ and risk of coronary disease, stroke, and mortality: Collaborative analysis of 32 prospective studies. Lancet 375 : $1536-1544,2010$

14. O'Donoghue M, Morrow DA, Sabatine MS, Murphy SA $\mathrm{McCabeCH}$,CannonCPandBraunwaldE:Lipoprotein-associated phospholipase A2 and its association with cardiovascular outcomes in patients with acute coronary syndromes in the PROVE IT-TIMI 22 (PRavastatin Or atorVastatin evaluation and infection therapy-thrombolysis in myocardial infarction) trial. Circulation 113: 1745-1752, 2006.

15. Albert MA, Glynn RJ, Wolfert RL and Ridker PM: The effect of statin therapy on lipoprotein associated phospholipase A2 levels. Atherosclerosis 182: 193-198, 2005.

16. White HD, Simes J, Stewart RA, Blankenberg S, Barnes EH, Marschner IC, Thompson P, West M, Zeller T, Colquhoun DM, et al: Changes in lipoprotein-associated phospholipase A2 activity predict coronary events and partly account for the treatment effect of pravastatin: Results from the long-term intervention with pravastatin in ischemic disease study. J Am Heart Assoc 2: e000360, 2013

17. Aydin MU, Aygul N, Altunkeser BB, Unlu A and Taner A: Comparative effects of high-dose atorvastatin versus moderate-dose rosuvastatin on lipid parameters, oxidized-LDL and inflammatory markers in ST elevation myocardial infarction. Atherosclerosis 239: 439-443, 2015.

18. Cannon CP, Blazing MA, Giugliano RP, McCagg A, White JA, Theroux P, Darius H, Lewis BS, Ophuis TO, Jukema JW, et al: Ezetimibe added to statin therapy after acute coronary syndromes. N Engl J Med 372: 2387-2397, 2015.

19. Davidson MH, Ballantyne CM, Jacobson TA, Bittner VA, Braun LT, Brown AS, Brown WV, Cromwell WC, Goldberg RB, McKenney JM, et al: Clinical utility of inflammatory markers and advanced lipoprotein testing: Advice from an expert panel of lipid specialists. J Clin Lipidol 5: 338-367, 2011.

20. Ballantyne CM, Hoogeveen RC, Raya JL, Cain VA, Palmer MK and Karlson BW; GRAVITY Study Investigators: Efficacy, safety and effect on biomarkers related to cholesterol and lipoprotein metabolism of rosuvastatin 10 or $20 \mathrm{mg}$ plus ezetimibe $10 \mathrm{mg}$ vs. simvastatin 40 or $80 \mathrm{mg}$ plus ezetimibe $10 \mathrm{mg}$ in high-risk patients: Results of the GRAVITY randomized study. Atherosclerosis 232: 86-93, 2014.
21. Moutzouri E, Liberopoulos EN, Tellis CC, Milionis HJ, Tselepis AD and Elisaf MS: Comparison of the effect of simvastatin versus simvastatin/ezetimibe versus rosuvastatin on markers of inflammation and oxidative stress in subjects with hypercholesterolemia. Atherosclerosis 231: 8-14, 2013.

22. Le NA, Tomassini JE, Tershakovec AM, Neff DR and Wilson PW: Effect of switching from statin monotherapy to ezetimibe/simvastatin combination therapy compared with other intensified lipid-lowering strategies on lipoprotein subclasses in diabetic patients with symptomatic cardiovascular disease. J Am Heart Assoc 4: e001675, 2015.

23. Song JX, Ren JY and Chen H: Simvastatin reduces lipoprotein-associated phospholipase A2 in lipopolysaccharide-stimulated human monocyte-derived macrophages through inhibition of the mevalonate-geranylgeranyl pyrophosphate-RhoA-p38 mitogen-activated protein kinase pathway. J Cardiovasc Pharmacol 57: 213-222, 2011.

24. Saougos VG, Tambaki AP, Kalogirou M, Kostapanos M, Gazi IF, Wolfert RL, Elisaf M and Tselepis AD: Differential effect of hypolipidemic drugs on lipoprotein-associated phospholipase A2. Arterioscler Thromb Vasc Biol 27: 2236-2243, 2007.

25. Zhang B, Fan P, Shimoji E, Itabe H, Miura S, Uehara Y, Matsunaga A and Saku K: Modulating effects of cholesterol feeding and simvastatin treatment on platelet-activating factor acetylhydrolase activity and lysophosphatidylcholine concentration. Atherosclerosis 186: 291-301, 2006.

26. Ostadal P, Vondrakova D, Kruger A, Janotka M, Psotova H and Prucha M: Alteration in lipoprotein-associated phospholipase A2 levels during acute coronary syndrome and its relationship to standard biomarkers. Lipids Health Dis 11: 153, 2012.

27. Li X, Kramer MC, van der Loos CM, Koch KT, de Boer OJ, Henriques JP, Baan J Jr, Vis MM, Piek JJ, Tijssen JG, et al: A pattern of disperse plaque microcalcifications identifies a subset of plaques with high inflammatory burden in patients with acute myocardial infarction. Atherosclerosis 218: 83-89, 2011.

28. Kawano Y, Narahara H and Johnston JM: Inhibitory effect of interleukin- 8 on the secretion of platelet-activating factor acetylhydrolase by human decidual macrophages. J Soc Gynecol Investig 6: 328-332, 1999.

29. Narahara H and Johnston JM: Effects of endotoxins and cytokines on the secretion of platelet-activating factor-acetylhydrolase by human decidual macrophages. Am J Obstet Gynecol 169: 531-537, 1993.

30. Lin ZP, Shu PC, Liao ZJ, Wang XQ and Liu Q: Rosuvastatin improves myocardial function and arteriosclerosis plaque in patients with ST-segment elevation after acute myocardial infarction and percutaneous coronary intervention. Nan Fang Yi Ke Da Xue Xue Bao 31: 1789-1791, 2011 (In Chinese).

31. Askevold ET, Gullestad L, Nymo S, Kjekshus J, Yndestad A, Latini R, Cleland JG, McMurray JJ, Aukrust P and Ueland T: Secreted frizzled related protein 3 in chronic heart failure: Analysis from the controlled rosuvastatin multinational trial in heart failure (CORONA). PLoS One 10: e0133970, 2015.

32. James SK, Oldgren J, Lindbäck J, Johnston N, Siegbahn A and Wallentin L: An acute inflammatory reaction induced by myocardial damage is superimposed on a chronic inflammation in unstable coronary artery disease. Am Heart J 149: 619-626, 2005.

33. Oldgren J, Wallentin L, Grip L, Linder R, Nørgaard BL and Siegbahn A: Myocardial damage, inflammation and thrombin inhibition in unstable coronary artery disease. Eur Heart J 24: 86-93, 2003.

34. Elkind MS, Leon V, Moon YP, Paik MC and Sacco RL: High-sensitivity C-reactive protein and lipoprotein-associated phospholipase A2 stability before and after stroke and myocardial infarction. Stroke 40: 3233-3237, 2009. 